\title{
LIMITING BEHAVIOR FOR AN ITERATED VISCOSITY*
}

\author{
Ciprian Foias, Michael S. Jolly ${ }^{1}$ and Oscar P. Manley ${ }^{2}$
}

\begin{abstract}
The behavior of an ordinary differential equation for the low wave number velocity mode is analyzed. This equation was derived in [5] by an iterative process on the two-dimensional NavierStokes equations (NSE). It resembles the NSE in form, except that the kinematic viscosity is replaced by an iterated viscosity which is a partial sum, dependent on the low-mode velocity. The convergence of this sum as the number of iterations is taken to be arbitrarily large is explored. This leads to a limiting dynamical system which displays several unusual mathematical features.
\end{abstract}

Mathematics Subject Classification. 35Q30, 37L65.

Received: December 22, 1999.

\section{INTRODUCTION}

The quest to find a closed form model of turbulence, started long ago (see e.g. $[10,11]$ ) and has involved many different approaches. None have succeeded. One of the more recent efforts amounts to finding an inertial manifold for the 2-D Navier-Stokes equations. To date, however, this equation lies just beyond the reach of the current existence results for inertial manifolds. There is however, a certain iterative process introduced in [5], which leads to a finite system of ordinary differential equations that while not necessarily equivalent to the Navier-Stokes equations, does enjoy many of the same mathematical properties. The process uses an approximate inertial manifold first considered in [4], to eliminate successive portions of the high wave number range of the spectrum of turbulent fluid flow. This system differs from most based on approximate inertial manifolds, in that an increase in the number of iterations does not further complicate the convective term, but rather adds to a series that forms a certain iterated viscosity. While it was shown in [6] that this system is dissipative for any finite number of iterations, until now the behavior as the number of iterations is taken to be arbitrarily large has not been analyzed. We show in this work that even though the iterated viscosity may not be bounded everywhere as the number of iterations is increased, we can still make sense of a limiting dynamical system.

To be slightly more specific, the system which is derived from the Navier-Stokes equations in two space dimensions with periodic boundary conditions, amounts to a differential equation for the low wave number mode $y_{1}$ with an iterated viscosity $\nu_{1}^{(n)}\left(y_{1}\right)$, where $n$ is the number of iterations. In [5] an exact form for the differential equation for $y_{1}$ is presented in which both the iterated viscosity $\nu_{1}^{(n)}\left(y_{1}\right)$ and nonlinear terms have been greatly simplified. These simplifications are mainly due to the spectral range of certain projections

\footnotetext{
Keywords and phrases. Navier-Stokes.

* Dedicated to Roger Temam for his 60th birthday

1 Department of Mathematics, Indiana University, Bloomington, IN, 47405, USA. e-mail: msjolly@indiana.edu

2 Gaithersburg, MD, 20878, USA.
} 
associated with the approximate inertial manifolds. Their proof is provided in [6], while the possible physical interpretations of some of the mathematical features of the system were discussed in [5]. From the physical point of view, the process that generates the equation for $y_{1}$ can be interpreted as following the successive decay of large turbulent eddies into smaller and smaller ones, as conceived in the classical picture of cascading turbulence. The iterated addition of the higher and higher spectral bands serves to generate terms which are readily recognized as contributions to the iterated viscosity in the equation for the low wavenumber velocity field.

In this paper we estimate the iterated viscosity in terms of the data for a given flow (e.g. Grashof number, etc.). We introduce a time-average of $\nu_{1}^{(n)}\left(y_{1}\right)$ as an effective viscosity for which we can obtain a sharper estimate. We then use this effective viscosity and the maximal ergodic theorem to obtain a statistical estimate for $\nu_{1}^{(n)}\left(y_{1}\right)$ on the global attractor $\mathcal{A}_{1}^{(n)}$ for the equation for $y_{1}$ after $n$ iterations. The dissipativity of the equation for $y_{1}$, is uniform in $n$, in that there is a common absorbing ball of a certain radius $R_{1}$. At the same time we can derive uniform bounds on $\nu_{1}^{(n)}\left(y_{1}\right)$ (independent of $n$ ) in a ball of a certain radius $r_{1}$. In order to make $r_{1} \geq R_{1}$, however, our estimates would require that $y_{1}$ extend deep into the dissipative range [9].

To be worthwhile, we insist that $y_{1}$ be limited to modes of much lower energy. This opens the possibility of a singular set $\mathcal{S}$, consisting of all $y_{1}$ such that $\nu_{1}^{(n)}\left(y_{1}\right) \rightarrow \infty$. Nevertheless we show that in the limit the iterative process provides a dynamical system with hitherto unrecognized properties which may be of more general mathematical interest. Any orbit passing through $\mathcal{S}$ does so along a trajectory of a purely linear system, instantaneously in time, and perhaps losing uniqueness both upon entry (in the past) and exit (in the future). Despite the possible lack of uniqueness, the global attractor $\mathcal{A}_{1}^{(\infty)}$ of the limiting system, defined to consist of all solutions which exist and are bounded for all time, makes sense. We find then that $\left\{\mathcal{A}_{1}^{(n)}\right\}$ is upper semicontinuous, as any neighborhood of $\mathcal{A}_{1}^{(\infty)}$ contains $\mathcal{A}_{1}^{(n)}$ for large enough $n$. This limiting behavior is determined by considering the trajectories as a function of arclength.

In a broader sense, the analysis here sheds some light on the algebraic properties of iterations of the convective term in the Navier-Stokes equations, and implicitly raises some open questions on this topic. We plan to report on the physical scope of our results here in a future publication.

As should be clear from this introduction, this paper originates in joint work with Roger Temam, to whom we are connected through a long scientific collaboration. In all this time we have admired Roger for his great mathematical talent, sustained diligence, and sound scientific judgement; but above all, for his caring friendship. We dedicate this paper to him on the occasion of his sixtieth birthday, with our best wishes for the continuation of his ascending scientific career.

\section{Preliminaries}

The periodic, incompressible Navier-Stokes equations

$$
\begin{aligned}
\frac{\partial u}{\partial t}-\nu \Delta u+(u \cdot \nabla) u+\nabla p & =F, \\
\operatorname{div} u & =0 \\
\int_{\Omega} u \mathrm{~d} x & =0 \\
u(x, 0) & =u_{0}(x)
\end{aligned}
$$

in $\Omega=[0, L]^{2}$ can be written as a differential equation in a certain Hilbert space $H$ (see [1] or [12]),

$$
\frac{\mathrm{d} u}{\mathrm{~d} t}+\nu A u+B(u, u)=f, \quad u \in H
$$


In fact $H$ is the domain in $L^{2}(\Omega)^{2}$ of the space of all $\mathbb{R}^{2}$-valued trigonometric polynomials $u$ such that

$$
\nabla \cdot u=0, \quad \text { and } \quad \int_{\Omega} u(x) \mathrm{d} x=0 .
$$

The scalar product in $H$ is defined by

$$
(u, v)=\int_{\Omega} u(x) \cdot v(x) \mathrm{d} x, \quad \text { where } \quad a \cdot b=a_{1} b_{1}+a_{2} b_{2},
$$

and the norm in $H$ is

Recall that $A=-\Delta$ defined on

$$
|u|=(u, u)^{1 / 2}=\left(\int_{\Omega} u(x) \cdot u(x) \mathrm{d} x\right)^{1 / 2} .
$$

$$
\mathcal{D}_{A}=\{u \in H:-\Delta u \in H\} .
$$

This operator is self-adjoint and its eigenvalues are of the form

$$
\left(\frac{2 \pi}{L}\right)^{2} k \cdot k, \quad \text { where } k \in \mathbb{Z}^{2} \backslash\{0\} .
$$

We denote by $0<\lambda_{0} \leq \lambda_{1} \leq \cdots$ these eigenvalues arranged in an increasing order and counted according to their multiplicities, and write $w_{0}, w_{1}, w_{2}, \ldots$, for the corresponding eigenvectors.

Clearly the positive square root of $A$ is defined by linearity from

$$
A^{1 / 2} w_{j}=\lambda_{j}^{1 / 2} w_{j}, \quad \text { for } j=0,1,2, \ldots
$$

on the set

$$
\mathcal{D}_{A^{1 / 2}}=\left\{u \in H: \sum_{j=0}^{\infty} \lambda_{j}\left(u, w_{j}\right)^{2}<\infty\right\} .
$$

Following an old tradition we will write $V=\mathcal{D}_{A^{1 / 2}}$ and the natural norm on $V$ will be

$$
\|u\|=\left|A^{1 / 2} u\right|=\left(\int_{\Omega} \sum_{j=1}^{2} \frac{\partial}{\partial x_{j}} u(x) \cdot \frac{\partial}{\partial x_{j}} u(x) \mathrm{d} x\right)^{1 / 2} .
$$

We define the projectors

$$
P(\lambda): H \rightarrow \operatorname{span}\left\{w_{j} \mid \lambda_{j} \leq \lambda\right\} .
$$

It is assumed that all the energy in the body force $f$ is concentrated in a certain number of modes with low eigenvalues so that

$$
P\left(\lambda_{f}\right) f=f, \text { for some } \lambda_{f}<\infty .
$$

Consider for the moment, fixing some wavenumber $\kappa_{1}>\lambda_{f}^{1 / 2}$. We set

$$
\kappa_{j}=2^{j-1} \kappa_{1}, \kappa_{0}=\lambda_{0}^{1 / 2}=2 \pi / L
$$

and henceforth index the projectors as

$$
P_{j}=P\left(\kappa_{j}^{2}\right), Q_{j}=I-P_{j} .
$$

Consider also a fixed $n \geq 1$, and set

$$
\nu_{n+1}=\nu, B_{n+1}=\left.B\right|_{P_{n+1} H},
$$


where $\nu$ is the kinematic viscosity and $B(u)=B(u, u)$ is the original bilinear term in (1.1)

$$
B(u, v)=\Pi[(u \cdot \nabla) v],
$$

where

$$
\varphi=\Pi \varphi+(I-\Pi) \varphi
$$

denotes the Helmholtz decomposition of a vector field into its divergence free component $(\operatorname{div} \Pi \varphi=0)$ and its gradient component.

For descending $j=n, n-1, \ldots, 1$ the following are recursively defined

$$
\begin{aligned}
\frac{\mathrm{d}}{\mathrm{d} t} y_{j}+\nu_{j}^{(n)}\left(y_{j}\right) A y_{j}+B_{j}\left(y_{j}\right) & =f, y_{j} \in P_{j} H \\
z_{j} & =-\left(\nu_{j+1}\left(y_{j}\right) A\right)^{-1} Q_{j} B_{j+1}\left(y_{j}\right) \\
\nu_{j}\left(y_{j}\right) & =\nu_{j+1}\left(y_{j}+z_{j}\right)+\nu_{j+1}\left(y_{j}\right) \frac{\left|A z_{j}\right|^{2}}{\left|A y_{j}\right|^{2}} \\
B_{j}\left(y_{j}\right) & =P_{j}\left[B_{j+1}\left(y_{j}\right)+B_{j+1}^{\prime}\left(y_{j}\right) z_{j}\right]-\nu_{j+1}\left(y_{j}\right) \frac{\left|A z_{j}\right|^{2}}{\left|A y_{j}\right|^{2}} A y_{j} .
\end{aligned}
$$

The reason for including the last term in the definition of $B_{j}$ is to preserve at each stage the orthogonality of the bilinear term with respect to $A y_{j}$, i.e.

$$
\left(B_{j}\left(y_{j}\right), A y_{j}\right)=0
$$

corresponding to

$$
(B(u, u), A u)=0
$$

for the bilinear term in the Navier-Stokes equations (for two different proofs of the latter, see [1] and [5]).

It was shown in [6] that the increase in the complexity of (1.2) through (1.5) with each successive iteration downward in $j$ is only apparent. In fact we have in those equations

$$
\nu_{j+1}\left(y_{j}\right)=\nu, B_{j+1}\left(y_{j}\right)=B\left(y_{j}\right), \quad \text { for } j=n, n-1, \ldots, 1 .
$$

In this paper we concentrate on the equation for the lowest modes, which thanks to (1.8) can be simply expressed as

$$
\frac{\mathrm{d} y_{1}}{\mathrm{~d} t}+\nu_{1}^{(n)}\left(y_{1}\right) A y_{1}+B_{1}\left(y_{1}\right)=f, \quad y_{1} \in P_{1} H
$$

with

$$
\nu_{1}^{(n)}\left(y_{1}\right)=\nu\left[1+\frac{\left|A z_{1}\right|^{2}}{\left|A y_{1}\right|^{2}}+\cdots+\frac{\left|A z_{n}\right|^{2}}{\left|A y_{n}\right|^{2}}\right]
$$

and

$$
B_{1}\left(y_{1}\right)=P_{1}\left[B\left(y_{1}\right)+B^{\prime}\left(y_{1}\right) z_{1}\right]-\nu \frac{\left|A z_{1}\right|^{2}}{\left|A y_{1}\right|^{2}} A y_{1}
$$


where we may now define in ascending order

$$
\begin{aligned}
& z_{1}=-(\nu A)^{-1} Q_{1} B\left(y_{1}, y_{1}\right), y_{2}=y_{1}+z_{1} \\
& z_{2}=-(\nu A)^{-1} Q_{2} B\left(y_{2}, y_{2}\right), y_{3}=y_{2}+z_{2} \\
& \vdots \\
& z_{n}=-(\nu A)^{-1} Q_{n} B\left(y_{n}, y_{n}\right), y_{n+1}=y_{n}+z_{n} .
\end{aligned}
$$

Note the change in notation: henceforth $y_{j}$, for $j=2,3, \ldots, y_{n+1}$ shall be defined as in (1.11) and are no longer taken to be the solutions to the differential equations in (1.2). Note also that the last term in $B_{1}\left(y_{1}\right)$ can be cancelled using the second term in $\nu_{1}^{(n)}$ to write the equation for $y_{1}$ simply as

$$
\frac{\mathrm{d} y_{1}}{\mathrm{~d} t}+\tilde{\nu}_{1}^{(n)}\left(y_{1}\right) A y_{1}+P_{1}\left[B\left(y_{1}\right)+B^{\prime}\left(y_{1}\right) z_{1}\right]=f
$$

where

$$
\tilde{\nu}_{1}^{(n)}\left(y_{1}\right)=\nu\left[1+\frac{\left|A z_{2}\right|^{2}}{\left|A y_{2}\right|^{2}}+\cdots+\frac{\left|A z_{n}\right|^{2}}{\left|A y_{n}\right|^{2}}\right] .
$$

We will use several other relations involving the bilinear term in the Navier-Stokes equations which are valid whenever all the implied operations are meaningful. Integrating

$$
(B(u, v), w)=\int \sum_{j, k=1}^{z} u_{j} \frac{\partial v_{\kappa}}{\partial x_{j}} w_{\kappa}
$$

by parts and applying the divergence free condition gives

$$
(B(u, v), w)=-(B(u, w), v)
$$

The bilinear term also enjoys the enstrophy invariance [8]

$$
(B(A v, v), u)-(B(v, A v), u)+(B(v, v), A u)=0 .
$$

We will also need Agmon's inequality in 2-D

$$
\|u\|_{\infty} \leq c_{1}|u|^{1 / 2}|A u|^{1 / 2}
$$

where the constant $c_{1}$ is a universal, and of the order of unity. If the spectrum of $u$ is in a wave number interval of the form $[\kappa, 2 \kappa]$, for some $\kappa>0$ then

where

$$
\|u\|_{\infty} \leq c_{1} \sqrt{2}\|u\|
$$

$$
\|u\|_{\infty}=\sup _{x} \sqrt{u(x) \cdot u(x)} .
$$

\section{An Estimate FOR $\nu_{1}^{(n)}$}

We begin by considering the rate of change of enstrophy associated with the low wave number velocity field, $y_{1}$, which, thanks to (1.6), satisfies

$$
\frac{1}{2} \frac{\mathrm{d}\left\|y_{1}\right\|^{2}}{\mathrm{~d} t}+\nu_{1}^{(n)}\left(y_{1}\right)\left|A y_{1}\right|^{2}=\left(f, A y_{1}\right)
$$


The use of Schwarz, Poincaré and Young's inequalities leads to

$$
\frac{\mathrm{d}\left\|y_{1}\right\|^{2}}{\mathrm{~d} t}+\nu \kappa_{0}^{2}\left\|y_{1}\right\|^{2} \leq \frac{\mathrm{d}\left\|y_{1}\right\|^{2}}{\mathrm{~d} t}+\nu_{1}^{(n)}\left(y_{1}\right) \kappa_{0}^{2}\left\|y_{1}\right\|^{2} \leq \frac{|f|^{2}}{\nu_{1}^{(n)}\left(y_{1}\right)} \leq \frac{|f|^{2}}{\nu}
$$

so that the ball of radius

$$
R_{1}=\frac{|f|}{\nu \kappa_{0}}
$$

is absorbing in $V$. Thus for (1.9) there exists a global attractor $\mathcal{A}_{1}=\mathcal{A}_{1}^{(n)} \subset \mathcal{B}_{\|\cdot\|}\left(0, R_{1}\right)$.

Lemma 2.1.

$$
\left\|y_{j+1}\right\|^{2} \leq\left\|y_{1}\right\|^{2}\left[1+\rho_{1}\left\|y_{1}\right\|^{2} \sum_{i=1}^{j}\left(\rho_{1}\left\|y_{j}\right\|^{2}\right)^{i-1} \frac{i !}{2^{i(i-1)}}\right]
$$

where

$$
\rho_{1}=\frac{c_{2} \log \frac{\kappa_{1}}{\kappa_{0}}}{\nu^{2} \kappa_{1}^{2}}
$$

for a certain universal constant $c_{2}$.

Proof. We divide the interval between $\kappa_{0}$ and $\kappa_{1}$ into two equal parts, denoting the velocity field in the upper half by $z_{0}$, and in the lower half by $y_{0}$. Next subdivide the interval between $\kappa_{0}$ and $\kappa_{1} / 2$ into two equal parts, denoting the velocity field in the upper half by $z_{-1}$, and in the lower half by $y_{-1}$. Repeat this process of successive subdivisions for additional $p-1$ steps, with the remaining lower portion of the spectrum in the final step being designated as $z_{-p}$. Then for any $i$ we can think of $y_{i}$ as

$$
y_{i}=z_{-p}+\ldots+z_{-1}+z_{0}+z_{1} \ldots+z_{i-1}
$$

Choose $p$ such that

$$
\frac{\kappa_{1}}{2^{p}} \leq \kappa_{0} \leq \frac{\kappa_{1}}{2^{p-1}}
$$

Applying (1.16), we have

$$
\begin{aligned}
\left\|y_{i}\right\|_{L^{\infty}} & \leq \sum_{j=-p}^{i-1}\left\|z_{j}\right\|_{L^{\infty}} \\
& \leq c \sum_{j=-p}^{i-1}\left\|z_{j}\right\|^{1 / 2}\left|A z_{j}\right|^{1 / 2} \\
& \leq c \sum_{j=-p}^{i-1} \frac{1}{\kappa_{j}}\left\|z_{j}\right\|^{1 / 2} \kappa_{j+1}\left\|z_{j}\right\|^{1 / 2} \\
& \leq c \sqrt{2} \sum_{j=-p}^{i-1}\left\|z_{j}\right\| \leq c \sqrt{2}(i+p)^{1 / 2}\left\|y_{i}\right\| .
\end{aligned}
$$


For $i$ and $p$ both larger than 1 , we have $j+p<2 j p$, so that

$$
\left\|y_{i}\right\|_{\infty} \leq c i^{1 / 2}\left(\log \frac{\kappa_{1}}{\kappa_{0}}\right)^{1 / 2}\left\|y_{i}\right\|
$$

where $c$ denotes different absolute constants.

Decomposing the bilinear term and using the fact that $Q_{i} B\left(y_{i-1}, y_{i-1}\right)=0$, we may write

$$
\nu A z_{i}=-Q_{i}\left[B\left(y_{i-1}, z_{i-1}\right)+B\left(z_{i-1}, y_{i-1}\right)+B\left(z_{i-1}, z_{i-1}\right)\right]
$$

Applying (1.16) and (2.6) we have

$$
\begin{aligned}
\nu\left|A z_{i}\right| & \leq\left\|y_{i-1}\right\|_{\infty}\left\|z_{i-1}\right\|+c_{1}\left|z_{i-1}\right|^{1 / 2}\left|A z_{i-1}\right|^{1 / 2}\left\|y_{i-1}\right\|+c_{1}\left|z_{i-1}\right|^{1 / 2}\left|A z_{i-1}\right|^{1 / 2}\left\|z_{i-1}\right\| \\
& \leq c\left[i^{1 / 2}\left(\log \frac{\kappa_{1}}{\kappa_{0}}\right)^{1 / 2}\left\|y_{i-1}\right\|+\left\|y_{i-1}\right\|+\left\|z_{i-1}\right\|\right]\left\|z_{i-1}\right\| \\
& \leq c i^{1 / 2}\left(\log \frac{\kappa_{1}}{\kappa_{0}}\right)^{1 / 2}\left\|y_{i}\right\|\left\|z_{i-1}\right\| .
\end{aligned}
$$

Since the wave numbers of the $z_{i}$ are bounded by $\kappa_{i}$ we have

$$
\nu^{2} \kappa_{i}^{2}\left\|z_{i}\right\|^{2} \leq \nu^{2}\left|A z_{i}\right|^{2} \leq c^{2} i \log \frac{\kappa_{1}}{\kappa_{0}}\left\|y_{i}\right\|^{2}\left\|z_{i-1}\right\|^{2}
$$

so that

$$
\left\|z_{i}\right\|^{2} \leq \rho_{1} \frac{i\left\|y_{i}\right\|^{2}}{4^{i-1}}\left\|z_{i-1}\right\|^{2}
$$

We consider $1 \leq i \leq j \leq n+1$, and the fact that $\left\|y_{i}\right\| \leq\left\|y_{j}\right\|$ to obtain

$$
\left\|z_{i}\right\|^{2} \leq \rho_{1} \frac{i\left\|y_{j}\right\|^{2}}{4^{i-1}}\left\|z_{i-1}\right\|^{2}
$$

Repeated applications of (2.9) provides

$$
\begin{aligned}
\left\|z_{i}\right\|^{2} & \leq\left(\rho_{1}\left\|y_{j}\right\|^{2}\right)^{i-1} \frac{i !}{4^{(i-1)+\cdots+1}}\left\|z_{1}\right\|^{2} \\
& =\left(\rho_{1}\left\|y_{j}\right\|^{2}\right)^{i-1} \frac{i !}{2^{i(i-1)}}\left\|z_{1}\right\|^{2}
\end{aligned}
$$

Setting $i=1$ in (2.8) we find that

$$
\left\|z_{1}\right\|^{2} \leq \rho_{1}\left\|y_{1}\right\|^{2}\left\|z_{0}\right\|^{2} \leq \rho_{1}\left\|y_{1}\right\|^{4}
$$


Finally, by combining (2.10) with (2.11), we obtain

$$
\begin{aligned}
\left\|y_{j+1}\right\|^{2} & =\left\|y_{1}\right\|^{2}+\left\|z_{1}\right\|^{2}+\cdots+\left\|z_{j}\right\|^{2} \\
& \leq\left\|y_{1}\right\|^{2}+\left\|z_{1}\right\|^{2}+\left[\sum_{i=2}^{j}\left(\rho_{1}\left\|y_{j}\right\|^{2}\right)^{i-1} \frac{i !}{2^{i(i-1)}}\right]\left\|z_{1}\right\|^{2} \\
& \leq\left\|y_{1}\right\|^{2}\left[1+\rho_{1}\left\|y_{1}\right\|^{2} \sum_{i=1}^{j}\left(\rho_{1}\left\|y_{j}\right\|^{2}\right)^{i-1} \frac{i !}{2^{i(i-1)}}\right] .
\end{aligned}
$$

Remark 2.2. The derivation of (2.6) can be simplified by using the following estimate from [7]

$$
\|y\|_{\infty} \leq c\|y\|\left(\log \frac{|A y|^{2}}{\kappa_{0}^{4}|y|^{2}}+1\right)^{1 / 2}, \quad \forall y \in \mathcal{D}_{A} .
$$

The form of the estimate in (2.3) suggests that we study the summation

$$
g(\xi)=\xi \sum_{i=1}^{\infty} \frac{i ! \xi^{i-1}}{2^{(i-1)^{2}}}, \quad \text { for } \xi \geq 0
$$

Applying the root test we find that

$$
0 \leq\left(\frac{n ! \xi^{n-1}}{2^{(n-1)^{2}}}\right)^{\frac{1}{n}} \leq \frac{n \xi^{1-\frac{1}{n}}}{2^{\frac{(n-1)^{2}}{n}}} \rightarrow 0, \quad \text { as } n \rightarrow \infty,
$$

so that the summation converges absolutely to a continuous function $g$. Note that $g$ is increasing with $\xi$, that $g(0)=0$, and

$$
g(1)=1+\sum_{i=2}^{\infty} \frac{i !}{2^{(i-1)^{2}}}>1 .
$$

Thus there exists a unique $\bar{\xi} \in(0,1)$ such that $g(\bar{\xi})=1$. Numerical evaluation by interval bisection gives

$$
0.5819 \leq \bar{\xi} \leq 0.5820 \text {. }
$$

We now set

and rewrite (2.3) as

$$
\xi_{j}=\rho_{1}\left\|y_{j}\right\|^{2} \quad \text { for } j=1,2, \ldots
$$

$$
\xi_{j+1}=\rho_{1}\left\|y_{j+1}\right\|^{2} \leq \xi_{1}\left(1+\xi_{1} \sum_{i=1}^{\infty} \frac{\xi_{j}^{i-1} i !}{2^{i(i-1)}}\right) .
$$

Lemma 2.3. If $\xi_{1} \leq \bar{\xi}$ then $\xi_{j} \leq \xi_{1}\left(1+g\left(\xi_{1}\right)\right) \leq 2 \xi_{1} \quad \forall j=1,2, \ldots$

Proof. We proceed by induction. Assume $\xi_{j} \leq 2 \xi_{1}$. It follows that

$$
\xi_{j+1} \leq \xi_{1}\left(1+\xi_{1} \sum_{i=1}^{\infty} \frac{\left(2 \xi_{1}\right)^{i-1} i !}{2^{i(i-1)}}\right)=\xi_{1}\left(1+g\left(\xi_{1}\right)\right) \leq \xi_{1}(1+g(\bar{\xi}))=2 \xi_{1} .
$$

We immediately have the following. 
Theorem 2.4. If $\rho_{1}\left\|y_{1}\right\|^{2} \leq \bar{\xi}$ then $\left\|y_{n}\right\|^{2} \leq 2\left\|y_{1}\right\|^{2}$ for $n=2,3, \ldots$

We now define

$$
\mathcal{D}_{\infty}=\left\{y_{1} \in P_{1} V:\left\|y_{1}\right\|^{2}+\left\|z_{1}\right\|^{2}+\left\|z_{2}\right\|^{2}+\ldots<\infty\right\}
$$

so that for each $y_{1} \in \mathcal{D}_{\infty}$ we have that

$$
y_{\infty}=y_{\infty}\left(y_{1}\right)=y_{1}+z_{1}+z_{2}+\ldots
$$

exists in $V$. Note that Theorem 2.4 states that if

$$
\left\|y_{1}\right\| \leq r_{1}=\sqrt{\frac{\bar{\xi}}{\rho_{1}}}=\frac{\bar{\xi}^{1 / 2} \nu \kappa_{1}}{c_{2}\left(\log \frac{\kappa_{1}}{\kappa_{0}}\right)^{1 / 2}}
$$

then $y_{1} \in \mathcal{D}_{\infty}$ and $\left\|y_{\infty}\right\|^{2} \leq 2\left\|y_{1}\right\|^{2}$.

Remark 2.5. Note that the condition $r_{1} \geq R_{1}$ is equivalent to

$$
\frac{\bar{\xi}}{c_{2}\left(\log \frac{\kappa_{1}}{\kappa_{0}}\right)^{1 / 2}} \frac{\kappa_{1}}{\kappa_{0}} \geq \frac{|f|}{\nu^{2} \kappa_{0}^{2}}=G
$$

where $G$ is the generalized Grashof number (see for example [3]). Thus, in order for our estimates to guarantee that $\mathcal{A}_{1}^{(n)} \subset \mathcal{D}_{\infty}$ we would need to take

$$
\frac{\kappa_{1}}{\kappa_{0}}=O(G)=O\left(\left(\frac{\kappa_{\eta}}{\kappa_{0}}\right)^{3}\right)
$$

where $\kappa_{\eta}$ is Kraichnan's dissipation wave number, beyond which no mode is expected to play any role in the dynamics of turbulent flow $[3,9]$. The condition (2.19) is then overly restrictive as it puts $\kappa_{1}$ deep into the so-called dissipative range.

We proceed to estimate $\nu_{1}^{(n)}$ for $y_{1} \in \mathcal{A}_{1}^{(n)} \cap \mathcal{B}_{\|\cdot\|}\left(0, r_{1}\right)$, regardless of whether $(2.19)$ holds.

Lemma 2.6. There is a universal constant $c_{3}$ such that

$$
\nu \frac{\left|A z_{j}\right|^{2}}{\left|A y_{j}\right|^{2}} \leq \frac{c_{3}}{\nu \kappa_{0}^{2}}\left\|z_{j-1}\right\|^{2}, \quad \forall y_{1} \in \mathcal{A}_{1}^{(n)}
$$

Proof. For convenience we define

$$
y_{0}=P\left(\frac{\kappa_{1}^{2}}{4}\right) y_{1}, z_{0}=Q\left(\frac{\kappa_{1}^{2}}{4}\right) y_{1}=\left(I-P\left(\frac{\kappa_{1}^{2}}{4}\right)\right) y_{1}
$$

We then have for all $j \geq 1$

$$
\nu A z_{j}=-Q_{j} B\left(y_{j}, y_{j}\right)=-Q_{j}\left[B\left(y_{j-1}, z_{j-1}\right)+B\left(z_{j-1}, y_{j-1}\right)+B\left(z_{j-1}, z_{j-1}\right)\right]
$$


By (1.16) we have

$$
\begin{aligned}
\nu\left|A z_{j}\right| \leq & \left|B\left(y_{j-1}, z_{j-1}\right)+B\left(z_{j-1}, y_{j-1}\right)\right|+c_{1}\left|z_{j-1}\right|^{1 / 2}\left|A z_{j-1}\right|^{1 / 2}\left|A^{1 / 2} z_{j-1}\right| \\
\leq & \left|B\left(y_{j-1}, z_{j-1}\right)+B\left(z_{j-1}, y_{j-1}\right)\right|+\sqrt{2} c_{1}\left|z_{j-1}\right|\left|A z_{j-1}\right| \\
\leq & c_{1}\left|y_{j-1}\right|^{1 / 2}\left|A y_{j-1}\right|^{1 / 2}\left|A^{1 / 2} z_{j-1}\right|+c_{1}\left|z_{j-1}\right|^{1 / 2}\left|A z_{j-1}\right|^{1 / 2}\left|A^{1 / 2} y_{j-1}\right| \\
& +c\left|z_{j-1}\right|\left|A z_{j-1}\right| \\
\leq & \frac{c}{\kappa_{0}}\left|A y_{j-1}\right|\left|A^{1 / 2} z_{j-1}\right|+\frac{c}{\kappa_{0}}\left|A y_{j-1}\right|\left|A^{1 / 2} z_{j-1}\right|+\frac{c}{\kappa_{0}}\left|A^{1 / 2} z_{j-1}\right|\left|A z_{j-1}\right| \\
\leq & \frac{c}{\kappa_{0}}\left|A^{1 / 2} z_{j-1}\right|\left|A y_{j}\right| .
\end{aligned}
$$

Lemma 2.7. For all $y_{1} \in \mathcal{A}_{1}^{(n)}$

$$
\nu_{1}^{(n)}\left(y_{1}\right) \leq \nu+\nu \xi_{1}+\nu\left(\frac{\kappa_{1}}{\kappa_{0}}\right)^{2} \frac{1}{\log \frac{\kappa_{1}}{\kappa_{0}}}\left[\sum_{i=1}^{\infty} \xi_{n+1}^{i-1} \frac{i !}{2^{i(i-1)}}\right] \xi_{1}^{2} .
$$

Proof. Expanding the bilinear term, then applying (1.15) and (1.14) we have

$$
\begin{aligned}
\nu\left|A z_{1}\right|^{2} & =-\left(B\left(y_{1}, y_{1}\right), A z_{1}\right)=\left(B\left(A y_{1}, y_{1}\right), z_{1}\right)-\left(B\left(y_{1}, A y_{1}\right), z_{1}\right) \\
& =\left(B\left(A y_{1}, y_{1}\right), z_{1}\right)+\left(B\left(y_{1}, z_{1}\right), A y_{1}\right) \leq c\left|A y_{1}\right|\left\|y_{1}\right\|\left(\log \frac{\kappa_{1}}{\kappa_{0}}\right)^{1 / 2}\left\|z_{1}\right\|
\end{aligned}
$$

Again since the spectrum of $z_{1}$ is bounded by $2 \kappa_{1}$, we have

which can be reexpressed as

$$
\nu\left|A z_{1}\right| \leq c\left\|y_{1}\right\|\left|A y_{1}\right| \frac{1}{\kappa_{1}}\left(\log \frac{\kappa_{1}}{\kappa_{0}}\right)^{1 / 2},
$$

By Lemma 2.6 we have

$$
\frac{\left|A z_{1}\right|^{2}}{\left|A y_{1}\right|^{2}} \leq \rho_{1}\left\|y_{1}\right\|^{2}
$$

$$
\nu_{j}^{(n)}\left(y_{j}\right)-\nu \leq \frac{c_{3}}{\nu \kappa_{0}^{2}}\left(\left\|z_{j-1}\right\|^{2}+\cdots+\left\|z_{n}\right\|^{2}\right)
$$

whence

$$
\nu_{1}^{(n)}\left(y_{1}\right) \leq \nu+\nu \rho_{1}\left\|y_{1}\right\|^{2}+\frac{c_{3}}{\nu \kappa_{0}^{2}}\left(\left\|z_{1}\right\|^{2}+\cdots+\left\|z_{n}\right\|^{2}\right)
$$

As in (2.12) we may estimate the contribution of the $z$-components as

$$
\begin{aligned}
\left\|z_{1}\right\|^{2}+\cdots+\left\|z_{n}\right\|^{2} & \leq\left[\sum_{i=1}^{n}\left(\rho_{1}\left\|y_{n+1}\right\|^{2}\right)^{i-1} \frac{i !}{2^{i(i-1)}}\right]\left\|z_{1}\right\|^{2} \\
& \leq\left[\sum_{i=1}^{\infty}\left(\rho_{1}\left\|y_{n+1}\right\|^{2}\right)^{i-1} \frac{i !}{2^{i(i-1)}}\right] \rho_{1}\left\|y_{1}\right\|^{4} .
\end{aligned}
$$

Substitution of this last bound into (2.8) completes the proof (using $c_{3} \leq c_{2}$ ). 
Theorem 2.8. For all $y_{1} \in \mathcal{A}_{1}^{(n)}$

$$
\xi_{1}=\rho_{1}\left\|y_{1}\right\|^{2} \leq \bar{\xi} \Rightarrow \nu_{1}^{(n)}\left(y_{1}\right) \leq \bar{\nu}^{0}=\nu+\nu \xi_{1}\left(1+\left(\frac{\kappa_{1}}{\kappa_{0}}\right)^{2} \frac{1}{\log \frac{\kappa_{1}}{\kappa_{0}}}\right)
$$

Thus if $\left\|y_{1}\right\| \leq r_{1}$, then $\nu_{1}^{(n)}\left(y_{1}\right) \leq \bar{\nu}^{0}$, for all $n \in \mathbb{N}^{+}$.

Proof. As in the proof of Lemma 2.3, we have

$$
\xi_{1} \leq \bar{\xi} \Rightarrow \xi_{1}\left[\sum_{i=1}^{\infty} \xi_{n+1}^{i-1} \frac{i !}{2^{i(i-1)}}\right] \leq g\left(\xi_{1}\right) \leq g(\bar{\xi})=1
$$

It turns out that boundedness of $\nu_{1}^{(n)}$ as $n \rightarrow \infty$, is equivalent to that for $y_{n}$ as $n \rightarrow \infty$.

Proposition 2.9. For all $y_{1}$ in $P_{1} H, \nu_{1}^{(\infty)}\left(y_{1}\right)<\infty$ if and only if $y_{\infty}$ in (2.16) exists in $V$, where

$$
\nu_{1}^{(\infty)}=\nu\left[1+\sum_{j=1}^{\infty} \frac{\left|A z_{j}\right|^{2}}{\left|A y_{j}\right|^{2}}\right]
$$

In this case, $A^{1 / 2} y_{\infty}$ is also in $V$.

Proof. Observe that since $\log (1+w) \leq w$ for all $w>0$ we have

$$
\frac{\left|A y_{\infty}\right|^{2}}{\left|A y_{1}\right|^{2}}=\frac{\left|A y_{2}\right|^{2}}{\left|A y_{1}\right|^{2}} \frac{\left|A y_{3}\right|^{2}}{\left|A y_{2}\right|^{2}} \cdots=\left(1+\frac{\left|A z_{1}\right|^{2}}{\left|A y_{1}\right|^{2}}\right)\left(1+\frac{\left|A z_{2}\right|^{2}}{\left|A y_{2}\right|^{2}}\right) \cdots=\exp \left[\sum_{n=1}^{\infty} \log \left(1+\frac{\left|A z_{n}\right|^{2}}{\left|A y_{n}\right|^{2}}\right)\right] \leq e^{\frac{\nu_{1}^{(\infty)}}{\nu}} .
$$

We conclude that

$$
\left|A y_{\infty}\right|^{2} \leq\left|A y_{1}\right|^{2} e^{\frac{\nu_{1}^{(\infty)}}{\nu}}
$$

The converse follows immediately from Lemma 2.6.

Thus far the crudest estimate seems to have been made in passing from (2.8) to (2.9) where we replaced $\left\|y_{i}\right\|$ by $\left\|y_{j}\right\|$ for $i<j$. This led to the constant $\bar{\xi}$ which determined $r_{1}$. Since as explained in Remark 2.5 , the value of $r_{1}$ and hence $\bar{\xi}$ is critical to our analysis, we now compare the estimate made above using (2.9) to one obtained using a nonlinear difference relation for $\eta_{j}$ and $\zeta_{j}$ where $\left\|y_{j}\right\|^{2} \leq \eta_{j}$ and $\left\|z_{j}\right\|^{2} \leq \zeta_{j}$, for $j=1,2,3, \ldots$, which is obtained without using that replacement referred to above. We may take $\eta_{1}=\left\|y_{1}\right\|$ as given, and since

$$
\left\|y_{j}\right\|^{2}=\left\|y_{j-1}\right\|^{2}+\left\|z_{j-1}\right\|^{2}
$$

we may also take $\zeta_{0}=\eta_{1}$. According to (2.8), valid bounds are generated by the recursion relations

$$
\begin{gathered}
\zeta_{j}=\rho_{1} \varepsilon_{j} \eta_{j} \zeta_{j-1}, \\
\eta_{j}=\eta_{j-1}+\zeta_{j-1}, j \geq 1,
\end{gathered}
$$

where $\varepsilon_{j}=j / 4^{j-1}$. Eliminating $\eta$ terms we find that

$$
\frac{\zeta_{j}}{\rho_{1} \varepsilon_{j} \zeta_{j-1}}=\eta_{j}=\eta_{j-1}+\zeta_{j-1}=\frac{\zeta_{j-1}}{\rho_{1} \varepsilon_{j-1} \zeta_{j-2}}+\zeta_{j-1}
$$


which may be reexpressed as a nonlinear difference scheme with 2 steps

$$
\zeta_{j}=\zeta_{j-1}^{2}\left(\rho_{1} \varepsilon_{j}+\frac{\varepsilon_{j}}{\varepsilon_{j-1}} \frac{1}{\zeta_{j-2}}\right)
$$

and finally as a system

$$
\begin{gathered}
\left(\zeta_{j+1}, \zeta_{j+2}\right)=\left(\zeta_{j+1}, \zeta_{j+1}^{2}\left(\rho_{1} \varepsilon_{j+2}+\frac{\varepsilon_{j+2}}{\varepsilon_{j+1}} \frac{1}{\zeta_{j}}\right)\right) \\
\zeta_{0}=\left\|y_{1}\right\|^{2}, \zeta_{1}=\rho_{1} \varepsilon_{1} \zeta_{0}^{2}
\end{gathered}
$$

Let $\tilde{\zeta}_{j}=\rho_{1} \varepsilon_{j+1} \zeta_{j}$, so that

$$
\tilde{\zeta}_{1}=\rho_{1} \varepsilon_{2} \zeta_{1}=\frac{\varepsilon_{2}}{\varepsilon_{1}}\left(\rho_{1} \varepsilon_{1} \zeta_{0}\right)^{2}=\frac{1}{2} \tilde{\zeta}_{0}^{2}
$$

and

$$
\tilde{\zeta}_{j+2}=\rho_{1} \varepsilon_{j+3}\left(\frac{\zeta_{j+1}}{\rho_{1} \varepsilon_{j+2}}\right)^{2}\left(\rho_{1} \varepsilon_{j+2}+\frac{\varepsilon_{j+2}}{\varepsilon_{j+1}} \frac{\rho_{1} \varepsilon_{j+1}}{\tilde{\zeta}_{j}}\right) .
$$

The system is now rewritten as

$$
\begin{gathered}
\left(\tilde{\zeta}_{j+1}, \tilde{\zeta}_{j+2}\right)=\left(\tilde{\zeta}_{j+1}, \tilde{\varepsilon}_{j} \tilde{\zeta}_{j+1}^{2}\left(1+\frac{1}{\tilde{\zeta}_{j}}\right)\right), \\
\tilde{\zeta}_{0}=\rho_{1}\left\|y_{1}\right\|^{2}, \tilde{\zeta}_{1}=\frac{1}{2} \tilde{\zeta}_{0}^{2},
\end{gathered}
$$

where $\tilde{\varepsilon}_{j}=\varepsilon_{j+3} / \varepsilon_{j+2}=(j+3) /(4 j+8)$.

Note that if for some $j$, we have $\tilde{\zeta}_{j+1} \geq 4$, then

$$
\tilde{\zeta}_{j+2} \geq \frac{\tilde{\zeta}_{j+1}}{4} \tilde{\zeta}_{j+1}\left(1+\frac{1}{\tilde{\zeta}^{j}}\right) \geq \tilde{\zeta}_{j+1}\left(1+\frac{1}{\tilde{\zeta}^{j}}\right)>\tilde{\zeta}_{j+1}
$$

In particular if

$$
\rho_{1}\left\|y_{1}\right\|^{2} \geq 2 \sqrt{2}, \text { then } \tilde{\zeta}_{1} \geq 4
$$

and consequently the nonlinear difference equation estimate for $\left\|z_{j}\right\|^{2}$, blows up as

$$
\zeta_{n} \geq \frac{4^{j}}{\rho_{1} j}
$$

Comparing (2.30) to (2.17), we conclude that further study of the nonlinear difference relation could only yield a slightly more relaxed condition than that in (2.22). 


\section{Effective Viscosity}

Integrating

$$
\frac{\mathrm{d}\left\|y_{1}\right\|^{2}}{\mathrm{~d} t}+\nu_{1}^{(n)}\left(y_{1}\right) \kappa_{0}^{2}\left\|y_{1}\right\|^{2} \leq \frac{|f|^{2}}{\nu_{1}^{(n)}\left(y_{1}\right)}
$$

under the assumption that the initial transient occurred at some far distant past (or equivalently, that we are on the global attractor, $\mathcal{A}_{1}$, of the differential equation controlling $\left.y_{1}\right)$, then

$$
\left\|y_{1}\right\|^{2} \leq|f|^{2} \int_{-\infty}^{t} \exp \left[-\kappa_{0}^{2} \int_{s}^{t} \nu_{1}^{(n)}\left(y_{1}(\tau)\right) \mathrm{d} \tau\right] \frac{\mathrm{d} s}{\nu_{1}^{(n)}\left(y_{1}(s)\right)} .
$$

It is convenient to define a time averaged effective viscosity, $\nu_{\text {eff }}\left(y_{1}\right)$,

$$
\frac{1}{\nu_{\mathrm{eff}}^{(n)}\left(y_{1}\right)} \equiv \sup _{(-\infty \leq s \leq 0)} \frac{1}{|s|} \int_{s}^{0} \frac{\mathrm{d} \tau}{\nu_{1}^{(n)}\left(y_{1}(\tau)\right)}
$$

Note that we have

$$
\nu \leq \nu_{\mathrm{eff}}^{(n)} \leq \nu_{1}^{(n)}
$$

We also have the following upper bound on $\nu_{\mathrm{eff}}^{(n)}$.

Theorem 3.1. If

$$
\frac{\kappa_{1}}{\kappa_{0}} \geq \frac{\left(c_{2} G\right)^{1 / 3}}{\bar{\xi}^{1 / 6}}\left(\log \kappa_{1} / \kappa_{0}\right)^{1 / 2}
$$

then

$$
\nu_{\mathrm{eff}}^{(n)}\left(y_{1}\right) \leq \nu\left[1+\bar{\xi}\left(1+\left(\frac{\kappa_{1}}{\kappa_{0}}\right)^{2} \frac{1}{\log \kappa_{1} / \kappa_{0}}\right)\right], \quad \forall y_{1} \in \mathcal{A}_{1}
$$

where $G$ is as defined in (2.18), and $c_{2}$ as in (2.4).

It is significant that the condition in (3.3) is consistent (up to a logarithm) with that of the Kraichnan wavelength $G^{1 / 3} \sim \kappa_{\eta} / \kappa_{0}$, where $\kappa_{\eta}$ is as in Remark 2.5. The proof of Theorem 3.1 requires the following estimate for the global attractor. For simplicity, throughout the remainder of this section, we will drop the explicit reference to the number of iterations, $n$, when referring to $\mathcal{A}_{1}, \nu_{1}$, and $\nu_{\text {eff }}$

Lemma 3.2. For all $y_{1} \in \mathcal{A}_{1}$, we have

$$
\left\|y_{1}\right\| \leq \kappa_{0} G \frac{\nu^{2}}{\nu_{\mathrm{eff}}\left(y_{1}\right)}
$$

Proof. Observing that

$$
1=\left(\frac{1}{|s|} \int_{s}^{0} \mathrm{~d} \tau\right)^{2} \leq\left(\frac{1}{|s|} \int_{s}^{0} \frac{\mathrm{d} \tau}{\nu_{1}\left(y_{1}(\tau)\right)}\right)\left(\frac{1}{|s|} \int_{s}^{0} \nu_{1}\left(y_{1}(\tau)\right) \mathrm{d} \tau\right) \leq \frac{1}{\nu_{\mathrm{eff}}\left(y_{1}\right)}\left(\frac{1}{|s|} \int_{s}^{0} \nu_{1}\left(y_{1}(\tau)\right) \mathrm{d} \tau\right)
$$

and integrating by parts (twice), we estimate $y_{1} \in \mathcal{A}_{1}$ by 


$$
\begin{aligned}
\left\|y_{1}\right\|^{2} & \leq|f|^{2} \int_{-\infty}^{0} e^{-\kappa_{0}^{2}|s| \nu_{\text {eff }}\left(y_{1}\right)} \frac{\mathrm{d} s}{\nu_{1}\left(y_{1}(s)\right)} \\
& =-|f|^{2} \int_{-\infty}^{0} e^{\kappa_{0}^{2} \nu_{\text {eff }}\left(y_{1}\right) s}\left(\int_{s}^{0} \frac{d \tau}{\nu_{1}\left(y_{1}(\tau)\right)}\right)_{s}^{\prime} \mathrm{d} s \\
& =|f|^{2} \kappa_{0}^{2} \nu_{\mathrm{eff}}\left(y_{1}\right) \int_{-\infty}^{0} e^{\kappa_{0}^{2} \nu_{\mathrm{eff}}\left(y_{1}\right) s}|s|\left(\frac{1}{|s|} \int_{s}^{0} \frac{d \tau}{\nu_{1}\left(y_{1}(\tau)\right)}\right) \mathrm{d} s \\
& \leq|f|^{2} \kappa_{0}^{2} \int_{-\infty}^{0} e^{\kappa_{0}^{2} \nu_{\mathrm{eff}}\left(y_{1}\right) s}|s| \mathrm{d} s \\
& =-\frac{|f|^{2}}{\nu_{\mathrm{eff}}\left(y_{1}\right)} \int_{-\infty}^{0} s\left(e^{\kappa_{0}^{2} \nu_{\mathrm{eff}}\left(y_{1}\right) s}\right)_{s}^{\prime} \mathrm{d} s=\frac{|f|^{2}}{\kappa_{0}^{2} \nu_{\mathrm{eff}}\left(y_{1}\right)^{2}} .
\end{aligned}
$$

Proof of Theorem 3.1 Suppose that (3.4) fails to hold for some $y_{1} \in \mathcal{A}_{1}$. Then for this particular $y_{1}$ we have

$$
\frac{\nu}{\nu_{\mathrm{eff}}\left(y_{1}\right)}<\left[1+\bar{\xi}\left(1+\left(\frac{\kappa_{1}}{\kappa_{0}}\right)^{2} \frac{1}{\log \kappa_{1} / \kappa_{0}}\right)\right]^{-1} .
$$

By Lemma 3.2, Theorem 2.8, and (3.2), we have that

$$
\bar{\xi}^{1 / 2}<c_{2}\left(\log \kappa_{1} / \kappa_{0}\right)^{1 / 2} G \frac{\kappa_{0}}{\kappa_{1}} \frac{\nu}{\nu_{\text {eff }}\left(y_{1}\right)} .
$$

Substituting (3.5) into the last estimate, and rearranging, we arrive at

$$
\bar{\xi}^{1 / 2}\left[1+\bar{\xi}\left(1+\left(\frac{\kappa_{1}}{\kappa_{0}}\right)^{2} \frac{1}{\log \kappa_{1} / \kappa_{0}}\right)\right]<c_{2}\left(\log \kappa_{1} / \kappa_{0}\right)^{1 / 2} G \frac{\kappa_{0}}{\kappa_{1}} .
$$

So for $x=\kappa_{1} / \kappa_{0}>e$ we have that

$$
\frac{x^{3}}{(\log x)^{3 / 2}}<x\left[1+\bar{\xi}\left(1+x^{2} \frac{1}{\log x}\right)\right] \frac{1}{(\log x)^{1 / 2}}<\frac{c_{2}}{\bar{\xi}^{1 / 2}} G,
$$

and consequently that

$$
\left(\frac{\kappa_{1}}{\kappa_{0}}\right)^{3}<\frac{c_{2}}{\bar{\xi}^{1 / 2}} G\left(\log \kappa_{1} / \kappa_{0}\right)^{3 / 2}
$$

which contradicts (3.3).

We are now also in a position to prove the following bound for $\nu_{\text {eff }}$, which will be used later to obtain some supplemental statistical information on $\nu_{1}$

Theorem 3.3. For all $y_{1} \in \mathcal{A}_{1}$

$$
\frac{\nu_{\mathrm{eff}}\left(y_{1}\right)}{\nu} \leq \gamma
$$

where

$$
\gamma=\max \left\{c G \frac{\kappa_{0}}{\kappa_{1}} \log \frac{\kappa_{1}}{\kappa_{0}}, c G^{2 / 3}\left(\left(\frac{\kappa_{0}}{\kappa_{1}}\right)^{2} \log \frac{\kappa_{1}}{\kappa_{0}}+1\right)^{1 / 3}\right\} .
$$


Proof. We have either

$$
\frac{\nu_{\mathrm{eff}}\left(y_{1}\right)}{\nu}<c \bar{\xi}^{-1 / 2} G \frac{\kappa_{0}}{\kappa_{1}} \log \frac{\kappa_{1}}{\kappa_{0}}
$$

or

$$
\frac{\nu_{\mathrm{eff}}\left(y_{1}\right)}{\nu} \geq c \bar{\xi}^{-1 / 2} G \frac{\kappa_{0}}{\kappa_{1}} \log \frac{\kappa_{1}}{\kappa_{0}}
$$

In the case of (3.7), we have by Lemma 3.2 that

$$
\bar{\xi} \geq \frac{c^{2} \log \frac{\kappa_{1}}{\kappa_{0}}}{\nu^{2} \kappa_{1}^{2}} G^{2}\left(\frac{\nu}{\nu_{\mathrm{eff}}\left(y_{1}\right)}\right)^{2} \kappa_{0}^{2} \nu^{2} \geq \frac{c^{2} \log \frac{\kappa_{1}}{\kappa_{0}}}{\nu^{2} \kappa_{1}^{2}}\left\|y_{1}\right\|^{2} .
$$

Lemma 2.3 now gives $\left\|y_{n+1}\right\|^{2} \leq 2\left\|y_{1}\right\|^{2}$, and we may apply Theorm 2.8 to obtain

$$
\begin{aligned}
\nu_{\mathrm{eff}}\left(y_{1}\right) & \leq \nu_{1}\left(y_{1}\right) \leq \nu+\nu \frac{c^{2} \log \frac{\kappa_{1}}{\kappa_{0}}}{\nu^{2} \kappa_{1}^{2}}\left\|y_{1}\right\|^{2}\left(1+\left(\frac{\kappa_{1}}{\kappa_{0}}\right)^{2} \frac{1}{\log \frac{\kappa_{1}}{\kappa_{0}}}\right) \\
& \leq \nu+\nu c^{2} \frac{\log \frac{\kappa_{1}}{\kappa_{0}}}{\left(\kappa_{1} / \kappa_{0}\right)^{2}} G^{2}\left(\frac{\nu}{\nu_{\mathrm{eff}}\left(y_{1}\right)}\right)^{2}\left(1+\left(\frac{\kappa_{1}}{\kappa_{0}}\right)^{2} \frac{1}{\log \frac{\kappa_{1}}{\kappa_{0}}}\right) .
\end{aligned}
$$

Thus if we set $\xi=\frac{\nu_{\text {eff }}\left(y_{1}\right)}{\nu}$ then we have

$$
\xi \leq 1+c^{2}\left(\frac{\log \frac{\kappa_{1}}{\kappa_{0}}}{\left(\kappa_{1} / \kappa_{0}\right)^{2}}+1\right) G^{2} \xi^{-2}
$$

or equivalently

$$
\xi \leq c G^{2 / 3}\left(\left(\frac{\kappa_{0}}{\kappa_{1}}\right)^{2} \log \frac{\kappa_{1}}{\kappa_{0}}+1\right)^{1 / 3}
$$

We now have the following statistical estimate on $\nu_{1}$.

Theorem 3.4. For any invariant probability measure $\mu$ on $\mathcal{A}_{1}$, one has

$$
\mu\left(\left\{y_{1} \in \mathcal{A}_{1}: \frac{\nu_{1}\left(y_{1}\right)}{\nu} \leq 2 \gamma\right\}\right) \geq \frac{1}{2 \gamma},
$$

where $\gamma$ is as in Theorem 3.3.

In other words the probability that $\nu_{1}\left(y_{1}\right) \leq 2 \nu \max \left\{c G \frac{\kappa_{0}}{\kappa_{1}}, c G^{2 / 3}\right\}$ (with a logarithmic correction) is $\geq 1 / 2 \gamma$ regardless of the stationary statistical distribution describing the permanent turbulent behavior of the reduced equation (1.9).

Proof. The function $1 / \nu_{\text {eff }}\left(y_{1}\right)$ is the maximal ergodic function associated to $1 / \nu_{\text {eff }}\left(y_{1}\right)$ ), and consequently we can apply the maximal ergodic theorem. Specifically in the notation used in [2], Chapter VIII. 7.6, page 690, we take $T_{t} f\left(y_{1}^{0}\right)=f\left(y_{1}(-t)\right)$, where $y_{1}$ is the solution of (1.9) such that $y_{1}(0)=y_{1}^{0}, y_{1}^{0} \in \mathcal{A}_{1}$ and $f$ is an arbitrary element in $L^{1}(\mu)$ (not the body force as in (1.1)). to obtain for $\alpha>0$

$$
\alpha \mu\left(e^{*}(2 \alpha)\right) \leq \int_{e(\alpha)} \frac{1}{\nu_{1}\left(y_{1}\right)} \mu\left(\mathrm{d} y_{1}\right) \leq \frac{1}{\nu} \mu(e(\alpha)),
$$


where

$$
e^{*}(2 \alpha)=\left\{y_{1} \in \mathcal{A}_{1}: \frac{1}{\nu_{\mathrm{eff}}\left(y_{1}\right)}>2 \alpha\right\}=\left\{y_{1} \in \mathcal{A}_{1}: \nu_{\mathrm{eff}}\left(y_{1}\right)<\frac{1}{2 \alpha}\right\}
$$

and

$$
e(\alpha)=\left\{y_{1} \in \mathcal{A}_{1}: \frac{1}{\nu_{1}\left(y_{1}\right)}>\alpha\right\}=\left\{y_{1} \in \mathcal{A}_{1}: \nu_{1}\left(y_{1}\right)<\frac{1}{\alpha}\right\}
$$

Let

$$
\frac{1}{2 \alpha}=\nu \gamma
$$

Then by Theorem 3.3 we have that $e^{*}(2 \alpha)=\mathcal{A}_{1}$, which completes the proof.

\section{Local Estimates}

The main result of this section establishes that $\mathcal{D}_{\infty}$ in $(2.15)$ is an open set. In particular we will estimate for each $y_{1}^{0} \in \mathcal{D}_{\infty}$, the radius of a ball in $V$, centered at $y_{1}^{0}$, which lies within $\mathcal{D}_{\infty}$. The smallness of this radius will effectively block our subsequent efforts in Section 5 to prove that the limiting system as $n \rightarrow \infty$ is locally Lipschitz, thereby allowing for nonuniqueness of solutions.

We have from (2.8) that

$$
\left\|z_{j}\right\|^{2} \leq \rho \varepsilon^{j}\left\|y_{j}\right\|^{2}\left\|z_{j-1}\right\|^{2}, \quad j=2,3, \ldots
$$

for $\rho, \varepsilon$ satisfying

In fact, we may take $\varepsilon=1 / 3$ and

$$
\rho \varepsilon^{j} \geq \rho_{1} \frac{j}{4^{j-1}} .
$$

$$
\rho=\rho_{1} \max _{2 \leq j} j(4 / 3)^{1-j} .
$$

Lemma 4.1. For $y_{\infty}=y_{1}+z_{1}+z_{2}+\cdots$, we have that

$$
\left\|y_{\infty}\right\|^{2}=\left\|y_{1}\right\|^{2}+\left\|z_{1}\right\|^{2}+\left\|z_{2}\right\|^{2}+\cdots<\infty
$$

if and only if there exists $n_{0} \in \mathbb{N}^{+}$such that

$$
\left\|y_{n_{0}}\right\|^{2} \sqrt{\rho \varepsilon^{n_{0}}} \leq 1
$$

and

$$
\left(1+\sqrt{\rho \varepsilon^{n_{0}}}\right) \sqrt{\varepsilon}<1 .
$$

Proof. If (4.2) holds, then

and consequently we have that as $n \rightarrow \infty$

$$
\left\|y_{n}\right\|^{2} \leq\left\|y_{\infty}\right\|^{2}<\infty, \forall n \in \mathbb{N}^{+},
$$

$$
\left\|y_{n}\right\|^{2} \sqrt{\rho \varepsilon^{n}} \rightarrow 0 \text { and }\left(1+\sqrt{\rho \varepsilon^{n}}\right) \sqrt{\varepsilon} \rightarrow \sqrt{\varepsilon}<1
$$

from which (4.3) and (4.4) follow immediately.

To prove the converse, we first use (4.1) to write

$$
\begin{aligned}
\left\|y_{n_{0}+1}\right\|^{2} & =\left\|y_{n_{0}}\right\|^{2}+\left\|z_{n_{0}}\right\|^{2} \\
& \leq\left\|y_{n_{0}}\right\|^{2}+\rho \varepsilon^{n_{0}}\left\|y_{n_{0}}\right\|^{2}\left\|z_{n_{0}-1}\right\|^{2} \\
& \leq\left\|y_{n_{0}}\right\|^{2}+\rho \varepsilon^{n_{0}}\left\|y_{n_{0}}\right\|^{4},
\end{aligned}
$$


so that by (4.3) we have

$$
\left\|y_{n_{0}+1}\right\|^{2} \leq\left\|y_{n_{0}}\right\|^{2}\left(1+\sqrt{\rho \varepsilon^{n_{0}}}\right)
$$

Applying (4.4) we find that

$$
\begin{aligned}
\left\|y_{n_{0}+1}\right\|^{2} \sqrt{\rho \varepsilon^{n_{0}+1}} & \leq\left\|y_{n_{0}}\right\|^{2}\left(1+\sqrt{\rho \varepsilon^{n_{0}}}\right) \sqrt{\varepsilon} \sqrt{\rho \varepsilon^{n_{0}}} \\
& <\left\|y_{n_{0}}\right\|^{2} \sqrt{\rho \varepsilon^{n_{0}}} \leq 1
\end{aligned}
$$

that is, (4.3) holds with $n_{0}$ replaced by $n_{0}+1$, as does (4.5) and (trivially) the condition (4.4). Repeatedly applying (4.5) we find that for all $n>n_{0}$

$$
\begin{aligned}
\left\|y_{n}\right\|^{2} & \leq\left\|y_{n-1}\right\|^{2}\left(1+\sqrt{\rho \varepsilon^{n-1}}\right) \\
& \leq\left\|y_{n_{0}}\right\|^{2}\left(1+\sqrt{\rho \varepsilon^{n_{0}}}\right) \cdots\left(1+\sqrt{\rho \varepsilon^{n-1}}\right) \\
& =\left\|y_{n_{0}}\right\|^{2} \exp \left(\sum_{j=n_{0}}^{n-1} \log \left(1+\sqrt{\rho \varepsilon^{j}}\right)\right)
\end{aligned}
$$

so that

$$
\left\|y_{n}\right\|^{2} \leq\left\|y_{n_{0}}\right\|^{2} \exp \left(\sqrt{\rho} \sum_{j=n_{0}}^{n-1}(\sqrt{\varepsilon})^{j}\right), \forall n>n_{0} .
$$

The convergence of the geometric series completes the proof.

We will need the estimate in (4.6) in the more general setting of the following.

Lemma 4.2. If $\left\|y_{\infty}\left(y_{1}\right)\right\|<\infty$ then

$$
\left\|y_{\infty}\left(y_{1}\right)\right\|^{2} \leq\left\|y_{n_{1}}(y)\right\|^{2}\left(1+\sqrt{\rho \varepsilon^{n_{1}}}\right)\left(1+\sqrt{\rho \varepsilon^{n_{1}+1}}\right) \cdots \quad \forall n_{1} \geq n_{0},
$$

where $n_{0}$ satisfies (4.3) and (4.4), and moreover

$$
\left\|z_{n}\right\|^{2} \leq \frac{1}{\rho} \varepsilon^{\frac{\left(n-n_{\infty}\right)\left(n-n_{\infty}-1\right)}{2}-n_{\infty}}
$$

where $n_{\infty} \geq n_{0}$ satisfies

$$
\rho \varepsilon^{n_{\infty}}\left\|y_{\infty}\left(y_{1}\right)\right\|^{2} \leq 1
$$

Proof. In the proof of Lemma 4.1 we saw that if $\left\|y_{\infty}\left(y_{1}\right)\right\|<\infty$, then (4.3) holds with $n_{0}$ replaced by $n_{1}$ for all $n_{1} \geq n_{0}$. It follows that (4.6) holds with $n_{0}$ replaced by $n_{1}$, which is precisely (4.8).

From (4.1) we have, since $\left\|y_{n}\right\| \leq\left\|y_{\infty}\right\|$,

$$
\begin{aligned}
\left\|z_{j}\right\|^{2} & \leq \rho \varepsilon^{j}\left\|y_{\infty}\right\|^{2}\left\|z_{j-1}\right\|^{2} \\
& \leq \varepsilon^{j-n_{\infty}}\left\|z_{j-1}\right\|^{2}, \quad \forall j<n_{\infty} .
\end{aligned}
$$

Repeating this estimate, we find that for all $n>n_{\infty}$

$$
\left\|z_{n}\right\|^{2} \leq \varepsilon^{\left(n-n_{\infty}\right)+\left(n-n_{\infty}-1\right)+\cdots+1}\left\|z_{n_{\infty}}\right\|^{2} .
$$

Now (4.8) follows from $\left\|z_{n_{\infty}}\right\|<\left\|y_{\infty}\right\|$. 
Theorem 4.3. If $\left\|y_{\infty}\left(y_{1}^{0}\right)\right\|<\infty$, for some $Y_{1}^{0} \in P_{1} H$, then there exists $r>0$ such that

$$
\left\|y_{\infty}\left(y_{1}\right)\right\| \leq 2\left\|y_{\infty}\left(y_{1}^{0}\right)\right\|, \quad \forall y_{1} \in \overline{\mathcal{B}_{\|\cdot\|}\left(y_{1}^{0}, r\right)} .
$$

Proof. By Lemma 4.1 there exists $n_{0}=n_{0}\left(\left\|y_{\infty}\left(y_{1}^{0}\right)\right\|\right.$ such that both

$$
\left\|y_{n_{0}}\left(y_{1}^{0}\right)\right\|^{2} \sqrt{\rho \varepsilon^{n_{0}}} \leq \sqrt{\varepsilon}
$$

and (4.4) hold. Since $y_{n_{0}}$ is a polynomial in $y_{1}$, it is continuous, and so there exists $r=r\left(n_{0}\right)>0$ such that

$$
\left\|y_{n_{0}}\left(y_{1}\right)\right\|^{2} \leq \frac{1}{\sqrt{\varepsilon}}\left\|y_{n_{0}}\left(y_{1}^{0}\right)\right\|, \quad \forall y_{1} \in \overline{\mathcal{B}}_{\|\cdot\|}\left(y_{1}^{0}, r\right) .
$$

From (4.11) we then have that (4.3) holds for all $y_{1} \in \overline{\mathcal{B}_{\|\cdot\|}\left(y_{1}^{0}, r\right)}$. Applying now the converse in Lemma 4.1 we obtain $\left\|y_{\infty}\left(y_{1}\right)\right\|^{2}<\infty$ and moreover from Lemma 4.2

$$
\begin{aligned}
\left\|y_{\infty}\left(y_{1}\right)\right\|^{2} & \leq\left\|y_{n_{1}}\left(y_{1}\right)\right\|^{2}\left(1+\sqrt{\rho \varepsilon^{n_{1}}}\right)\left(1+\sqrt{\rho \varepsilon^{n_{1}+1}}\right) \ldots \\
& \leq\left\|y_{n_{1}}\left(y_{1}^{0}\right)\right\|^{2} \frac{1}{\sqrt{\varepsilon}}\left(1+\sqrt{\rho \varepsilon^{n_{1}}}\right)\left(1+\sqrt{\rho \varepsilon^{n_{1}+1}}\right) \ldots
\end{aligned}
$$

Taking $n_{1}$ large enough so that

$$
\left(1+\sqrt{\rho \varepsilon^{n_{1}}}\right)\left(1+\sqrt{\rho \varepsilon^{n_{1}+1}}\right) \cdots \leq 2 \sqrt{\varepsilon}
$$

completes the proof.

Remark 4.4. Thanks to (4.10) we may find $n_{\infty}$ for which (4.9) holds for all

$$
y_{1} \in \overline{\mathcal{B}_{\|\cdot\|}\left(y_{1}^{0}, r\right)} .
$$

\section{Differentiability OF $y_{\infty}$ AND $\nu_{1}^{(\infty)}$}

We will establish the local Lipschitz property for the limiting system at $n \rightarrow \infty$ on the set $\mathcal{D}_{\infty}$, by proving that on that set the stronger property of differentiability holds.

Theorem 5.1. For each $y_{1}^{0} \in \mathcal{D}_{\infty}$, the Frechet derivatives of both $y_{\infty}\left(y_{1}\right)$ and $\nu_{1}^{(\infty)}$ with respect to $y_{1}$ exist throughout $\overline{\mathcal{B}_{\|\cdot\|}\left(y_{1}^{0}, r\right)}$, where $r$ is as in Theorem 4.3 .

Proof. For each $j \in \mathbb{N}^{+}$let

$$
y_{j}^{\prime}=\frac{\mathrm{d}}{\mathrm{d} y_{1}} y_{j}\left(y_{1}\right)
$$

be the Frechet derivative of $y_{j}=y_{j}\left(y_{1}\right)$ evaluated at $y_{1}$. By (1.11) we have

$$
y_{n+1}^{\prime} \delta_{1}=y_{n}^{\prime} \delta_{1}-(\nu A)^{-1} Q_{n}\left[B\left(y_{n}, y_{n}^{\prime} \delta_{1}\right)+B\left(y_{n}^{\prime} \delta_{1}, y_{n}\right)\right], \quad \forall \delta_{1} \in P_{1} H .
$$

As in the derivation of (2.7) we bound the bilinear terms as

$$
\begin{aligned}
\left\|y_{n+1}^{\prime} \delta_{1}\right\|^{2} & =\left\|y_{n}^{\prime} \delta_{1}\right\|^{2}+\left\|(\nu A)^{-1} Q_{n}\left[B\left(y_{n}, y_{n}^{\prime} \delta_{1}\right)+B\left(y_{n}^{\prime} \delta_{1}, y_{n}\right)\right]\right\|^{2} \\
& \leq\left\|y_{n}^{\prime} \delta_{1}\right\|^{2}+2 \rho \varepsilon^{n}\left\|y_{n}\right\|^{2}\left\|y_{n}^{\prime} \delta_{1}\right\|^{2} \\
& =\left\|y_{n}^{\prime} \delta_{1}\right\|^{2}\left(1+2 \rho \varepsilon^{n}\left\|y_{n}\right\|^{2}\right) .
\end{aligned}
$$


Thanks to Theorem 4.3 we may choose $n_{2}>n_{\infty}$ such that

$$
\left\|y_{n}\left(y_{1}\right)\right\| \leq\left\|y_{\infty}\left(y_{1}\right)\right\| \leq\left\|y_{\infty}\left(y_{1}^{0}\right)\right\| \leq\left(2 \rho \varepsilon^{n_{2}}\right)^{-1 / 4}, \quad \forall y_{1} \in \overline{\mathcal{B}_{\|\cdot\|}\left(y_{1}^{0}, r\right)}
$$

Continuing the estimate for $\left\|y_{n+1}^{\prime} \delta_{1}\right\|^{2}$, we have

$$
\begin{aligned}
\left\|y_{n+1}^{\prime} \delta_{1}\right\|^{2} & \leq\left\|y_{n}^{\prime} \delta_{1}\right\|^{2}\left(1+\sqrt{2 \rho \varepsilon^{n}}\right) \\
& \leq\left\|y_{n_{2}}^{\prime} \delta_{1}\right\|^{2} \prod_{j=n_{2}}^{\infty}\left(1+\sqrt{2 \rho \varepsilon^{j}}\right) \\
& \leq\left\|y_{n_{2}}^{\prime} \delta_{1}\right\|^{2} \exp \left(\sqrt{2 \rho} \sum_{j=n_{2}}^{\infty} \varepsilon^{j / 2}\right) .
\end{aligned}
$$

By the convergence of the last geometric series, we have that $\left\|y_{n}^{\prime}\right\|$ is bounded, independently of $n$. Since

$$
\left\|y_{n}^{\prime}\right\|^{2}=\left\|y_{1}^{\prime}\right\|^{2}+\sum_{j=1}^{n}\left\|z_{j}^{\prime}\right\|^{2}
$$

it follows that $\left\{y_{n}^{\prime}\right\}_{n=1}^{\infty}$ is convergent, uniformly so on $\overline{\mathcal{B}_{\|\cdot\|}\left(y_{1}^{0}, r\right)}$ thanks to the fact that $n_{2}$ is independent of $y_{1}$. By a well-known result from classical analysis, which extends to the Frechet case, the uniform convergence of $\left\{y_{n}^{\prime}\right\}_{n=1}^{\infty}$, together with the convergence of $\left\{y_{n}\right\}_{n=1}^{\infty}$ at say $y_{1}^{0}$, implies that $y_{n}^{\prime} \rightarrow\left(y_{\infty}\right)^{\prime}$ on $\overline{\mathcal{B}_{\|\cdot\|}\left(y_{1}^{0}, r\right)}$.

We now turn to the differentiability of $\nu_{1}^{\infty}$. We proceed by estimating the derivative of each term in the summation with

$$
\begin{aligned}
\left|\left[\frac{\mathrm{d}}{\mathrm{d} y_{1}}\left(\frac{\left|A z_{n}\right|^{2}}{\left|A y_{n}\right|^{2}}\right)\right] \delta_{1}\right| & =\left|\frac{2\left(A z_{n},\left(A z_{n}^{\prime}\right) \delta_{1}\right)}{\left|A y_{n}\right|^{2}}-\frac{\left|A z_{n}\right|^{2}}{\left|A y_{n}\right|^{2}} \frac{2\left(A y_{n},\left(A y_{n}^{\prime}\right) \delta_{1}\right)}{\left|A y_{n}\right|^{2}}\right| \\
& \leq \frac{2\left|A^{3 / 2} z_{n}\right||| z_{n}^{\prime} \delta_{1} \|}{\left|A y_{n}\right|^{2}}+\frac{2\left|A z_{n}\right|^{2}}{\left|A y_{n}\right|^{2}} \frac{\left|A^{3 / 2} y_{n}\right|\left\|y_{n}^{\prime} \delta_{1}\right\|}{\left|A y_{n}\right|^{2}} \\
& \leq 2\left(\frac{\left|A^{3 / 2} z_{n}\right|^{2}}{\left|A y_{n}\right|^{4}}+\frac{\left|A^{3 / 2} z_{n}\right|^{4}}{\left|A y_{n}\right|^{8}}\left|A^{3 / 2} y_{n}\right|^{2}\right)^{1 / 2}\left(\left\|y_{n}^{\prime} \delta_{1}\right\|^{2}+\left\|z_{n}^{\prime} \delta_{1}\right\|^{2}\right)^{1 / 2}
\end{aligned}
$$

where we used the Cauchy-Schwarz inequality in $\mathbb{R}^{2}$,

$$
\left|a_{1} b_{1}+a_{2} b_{2}\right| \leq\left\{\left(a_{1}^{2}+a_{2}^{2}\right)\left(b_{1}^{2}+b_{2}^{2}\right)\right\}^{1 / 2}
$$

with

$$
a_{1}=\frac{\left|A^{3 / 2} z_{n}\right|}{\left|A y_{n}\right|^{2}}, \quad a_{2}=\frac{\left|A z_{n}\right|^{2}}{\left|A y_{n}\right|^{4}}\left|A^{3 / 2} y_{n}\right|, \quad b_{1}=\left\|z_{n}^{\prime} \delta_{1}\right\|, \quad b_{2}=\left\|y_{n}^{\prime} \delta_{1}\right\|
$$


Continuing the estimate, we have by (4.8) that

$$
\begin{aligned}
& \left|\left[\frac{\mathrm{d}}{\mathrm{d} y_{1}}\left(\frac{\left|A z_{n}\right|^{2}}{\left|A y_{n}\right|^{2}}\right)\right] \delta_{1}\right| \leq\left\|y_{n+1}^{\prime} \delta_{1}\right\|\left(\left(\frac{\kappa_{n+1}}{\kappa_{0} r_{1}}\right)^{4}\left\|z_{n}\right\|^{2}+\frac{\kappa_{n+1}^{12}}{\left(\kappa_{0} r\right)^{8}}\left\|z_{n}\right\|^{4}\right)^{1 / 2} \\
& \leq\left\|y_{n+1}^{\prime} \delta_{1}\right\|\left\{\left(\frac{2^{n} \kappa_{1}}{\kappa_{0} r_{1}}\right)^{4} \frac{1}{\rho} \varepsilon^{\frac{\left(n-n_{\infty}\right)\left(n-n_{\infty}-1\right)}{2}-n_{\infty}}+\frac{2^{12 n} \kappa_{1}^{12}}{\left(\kappa_{0} r_{1}\right)^{8} \rho^{2}} \varepsilon^{\left(n-n_{\infty}\right)\left(n-n_{\infty}-1\right)-2 n_{\infty}}\right\}^{1 / 2} \\
& \leq\left\|y_{n+1}^{\prime} \delta_{1}\right\| \frac{2^{6 n+1} \kappa_{1}^{6}}{\sqrt{\rho_{1}}\left(\kappa_{0} r_{1}\right)^{2}} \varepsilon^{\frac{\left(n-n_{\infty}\right)\left(n-n_{\infty}-1\right)}{4}-\frac{n_{\infty}}{2}} \\
& \leq\left\|y_{n+1}^{\prime} \delta_{1}\right\| C\left(n_{\infty}\right) 64^{n} \varepsilon^{\left(n-n_{\infty}\right)^{2}} .
\end{aligned}
$$

Thus

$$
\sum_{1}^{\infty}\left[\frac{\mathrm{d}}{\mathrm{d} y_{1}}\left(\frac{\left|A z_{n}\right|^{2}}{\left|A y_{n}\right|^{2}}\right)\right]
$$

is bounded in each component by a geometric series which is convergent, uniformly so, thanks to Remark 4.4. Now a similar argument to that for $y_{\infty}$ shows that $\left(\nu_{1}^{(n)}\right)^{\prime} \rightarrow\left(\nu_{1}^{(\infty)}\right)^{\prime}$ on $\overline{\mathcal{B}_{\|\cdot\|}\left(y_{1}^{0}, r\right)}$. Due to uniform convergence, both $y_{\infty}^{\prime}$ and $\left(\nu_{1}^{(\infty)}\right)^{\prime}$ are continuous.

Remark 5.2. In fact if $y_{1} \in \mathcal{D}_{\infty},\left\|w_{1}\right\|=1$ and $s \geq 0$ is small enough, then

$$
\frac{1}{2} \frac{\mathrm{d}}{\mathrm{d} s}\left\|y_{\infty}\left(y_{1}+s w_{1}\right)\right\|^{2}=\left(y_{\infty}\left(y_{1}+s w_{1}\right), y_{\infty}^{\prime}\left(y_{1}+s w_{1}\right) w_{1}\right) \leq\left\|y_{\infty}\left(y_{1}+s w_{1}\right)\right\|\left\|y_{\infty}^{\prime}\left(y_{1}+s w_{1}\right) w_{1}\right\|
$$

whence

$$
\frac{\mathrm{d}}{\mathrm{d} s}\left\|y_{\infty}\left(y_{1}+s w_{1}\right)\right\| \leq\left\|y_{\infty}^{\prime}\left(y_{1}+s w_{1}\right)\right\|
$$

Using (5.1), we can easily obtain a differential inequality for $\left\|y_{\infty}\left(y_{1}+s w_{1}\right)\right\|$ from which it follows that $\left\|y_{\infty}\left(y_{1}+s w_{1}\right)\right\|<\infty$ as long as

$$
s<c_{4} e^{-c_{5}\left(\log \left(\left\|y_{\infty}\left(y_{1}\right)\right\| \sqrt{2 \rho}\right)\right)^{2}}
$$

where $c_{4}, c_{5}$ are absolute constants $>0$.

\section{Limiting BeHAVior}

According to the mathematicians this series is divergent; therefore we may be able to do something useful with it.

-O. Heaviside [13]

Our analysis up to this point would suggest that the iteration presented earlier introduces no anomalies as it is continued to infinity. In fact we are not quite able to conclude this. As we demonstrate here there is a possibility of pathological behavior, such as $y_{1}^{(\infty)}$ becoming discontinuous with respect to time, while the iterated viscosity $\nu_{1}^{(\infty)}$ becomes momentarily infinite. The reason is that we are unable to show that $y_{n}\left(y_{1}\right)$, and $\nu_{1}^{(n)}\left(y_{1}\right)$ are bounded independent of $n$, for all $y_{1} \in P_{1} H$. Nevertheless, we are still able to make sense of the limiting dynamical system on all of $P_{1} H$ as $n \rightarrow \infty$. This is done by replacing the time parametrization by arclength parametrization. To do so, we first must isolate the steady states. 
We multiply the steady state equation for (1.9)

$$
\nu_{1}^{(n)} A y_{1}+B_{1}\left(y_{1}, y_{1}\right)=f
$$

by $A y_{1}$, integrate, and apply (1.7) to obtain

$$
\nu_{1}^{(n)}\left|A y_{1}\right|^{2}=\left(f, A y_{1}\right) \leq|f|\left|A y_{1}\right|<\frac{|f|^{2}}{2 \nu_{1}^{(n)}}+\nu_{1}^{(n)} \frac{\left|A y_{1}\right|^{2}}{2}
$$

so that

and hence

$$
\left|A y_{1}\right| \leq \frac{|f|}{\nu_{1}^{(n)}\left(y_{1}\right)}
$$

$$
\left\|y_{1}\right\| \leq \frac{|f|}{\kappa_{0} \nu_{1}^{(n)}\left(y_{1}\right)}=R_{1} \frac{\nu}{\nu_{1}^{(n)}\left(y_{1}\right)},
$$

where $R_{1}$ is as in (2.2). Let $\nu_{0}=R_{1} \nu / r_{1}$ with $r_{1}$ as in (2.17). If $\nu_{0} \leq \nu_{1}^{(n)}$, then

$$
\left\|y_{1}\right\| \leq R_{1} \frac{\nu}{\nu_{0}}=r_{1}
$$

and by Corollary 2.8 we have

$$
\nu_{1}^{(n)} \leq \bar{\nu}_{0}
$$

where $\bar{\nu}_{0}$ is as in (2.22).

It follows that at any steady state $y_{1}^{(n)}$

$$
\nu_{1}^{(n)}=\nu_{1}^{(n)}\left(y_{1}^{(n)}\right) \leq \max \left\{\nu_{0}, \bar{\nu}_{0}\right\}
$$

Let

$$
\mathcal{O}_{n}(\alpha)=\left\{y_{1} \in P_{1} H:\left\|y_{1}\right\|<R_{1}, \nu_{1}^{(n)}>\alpha \max \left(\nu_{0}, \bar{\nu}_{0}\right)\right\} .
$$

Note that for all $\alpha>1, \mathcal{O}_{n}(\alpha)$ does not contain any steady states of (1.9) and lies within the annulus

$$
D=\left\{y_{1} \in P_{1} H: r_{1}<\left\|y_{1}\right\|<R_{1}\right\}
$$

We now consider two sequences of differential equations, both of which are equivalent to (1.9) on overlapping domains

$$
\begin{gathered}
\frac{\mathrm{d}}{\mathrm{d} t} y_{1}=F_{n}\left(y_{1}\right)=f-B_{1}\left(y_{1}\right)-\nu_{1}^{(n)} A y_{1}, \quad y \in P_{1} H \backslash \mathcal{O}_{n}\left(2 \alpha_{n}\right) \\
\frac{\mathrm{d}}{\mathrm{d} s}\left(Y_{1}, t\right)=G_{n}\left(Y_{1}\right)=\left(\frac{F_{n}}{\left\|F_{n}\right\|}, \frac{1}{\left\|F_{n}\right\|}\right), \quad\left(Y_{1}, t\right) \in \mathcal{O}_{n}\left(\alpha^{*}\right) \times \mathbb{R},
\end{gathered}
$$

where $Y_{1}(s)=y_{1}(t(s))$, and $\alpha^{*},\left\{\alpha_{n}\right\}$ are to be determined.

We rewrite the first component of $G_{n}$ as

$$
\begin{aligned}
\frac{F_{n}\left(Y_{1}\right)}{\left\|F_{n}\left(Y_{1}\right)\right\|} & =\frac{1}{\nu_{1}^{(n)}}\left[f-B_{1}\left(Y_{1}\right)\right] \frac{1}{\left\|A Y_{1}+\frac{1}{\nu_{1}^{(n)}}\left[B_{1}\left(Y_{1}\right)-f\right]\right\|}-A Y_{1} \frac{1}{\left\|A Y_{1}+\frac{1}{\nu_{1}^{(n)}}\left[B_{1}\left(Y_{1}\right)-f\right]\right\|} \\
& =-\frac{A Y_{1}}{\left\|A Y_{1}\right\|}+E_{n}\left(Y_{1}\right) .
\end{aligned}
$$


By the compactness of $\bar{D}$ (the closure of $D$ ), there exists $\alpha^{*} \geq 1$ such that

$$
\left\|F_{n}\left(Y_{1}\right)\right\| \geq 1, \text { for all } Y_{1} \in \mathcal{O}_{n}\left(\alpha^{*}\right),
$$

and moreover there exists $\left\{\alpha_{n}\right\}$ such that

$$
\alpha_{n}>\alpha^{*} \text { and }\left\|E_{n}\left(Y_{1}\right)\right\|<1 / n \text {, for all } Y_{1} \in \mathcal{O}_{n}\left(\alpha_{n}\right) .
$$

Let $\mathcal{S}$ denote the (potentially empty) set in $D$ where the iterated viscosity grows without bound, i.e.

$$
\mathcal{S}=\left\{y_{1} \in P_{1} H: \lim _{n \rightarrow \infty} \nu_{1}^{(n)}\left(y_{1}\right)=\infty\right\}
$$

We write the limiting system as

$$
\frac{\mathrm{d} y_{1}}{\mathrm{~d} t}=f-B_{1}\left(y_{1}\right)-\nu_{1}^{(\infty)} A y_{1}, \quad y_{1} \in P_{1} H \backslash \bigcap_{n=1}^{\infty} \overline{\mathcal{O}_{n}\left(2 \alpha_{n}\right)}
$$

and

$$
\frac{\mathrm{d}}{\mathrm{d} s}\left(Y_{1}, t\right)= \begin{cases}\left(\frac{f-B_{1}\left(Y_{1}\right)-\nu_{1}^{(\infty)} A Y_{1}}{\left\|f-B_{1}\left(Y_{1}\right)-\nu_{1}^{(\infty)} A Y_{1}\right\|}, \frac{1}{\left\|f-B_{1}\left(Y_{1}\right)-\nu_{1}^{(\infty)} A Y_{1}\right\|}\right) & \text { if } Y_{1} \in \mathcal{O}\left(\alpha^{*}\right) \backslash \mathcal{S} \\ \left(-\frac{A Y_{1}}{\left\|A Y_{1}\right\|}, 0\right) & \text { if } Y_{1} \in \mathcal{S} .\end{cases}
$$

Multiplying (6.3) by $A y_{1}$, the first component in (6.4) by $A Y_{1}$, and proceeding as for (2.1) we find that the ball of radius $R_{1}$ is absorbing for the limiting system. We define the global attractor $\mathcal{A}_{1}^{(\infty)}$ of the limiting system as consisting of all solutions (just the first component, in the case of (6.4) which exist and are bounded for all $t \in \mathbb{R}$.

Since the Frechet derivative of $F_{n}$ is continuous, $F_{n}$ is locally Lipschitz throughout $P_{1} H$ for all $n \in \mathbb{N}^{+}$. On the other hand, even though $\nu_{1}^{(\infty)}$ is continuously differentiable wherever it is bounded, the most we can say about the vector field for the limiting system $(6.3,6.4)$ is that it is locally Lipschitz everywhere except at the boundary of the singular set $\mathcal{S}$. If we could improve the estimate sketched in Remark 5.2, this exception could be overcome.

By the continuous dependence of the solution of an ordinary differential equation on its vector field, we have that any sequence of integral curves of $(6.1,6.2)$ contains a subsequence which converges to an integral curve of $(6.3,6.4)$. On the boundary of $\mathcal{S}$ however, we may lose uniqueness of solutions, backward in time at a point of entry into $\mathcal{S}$, and forward in time at a point of exit from $\mathcal{S}$, as depicted in the figure below. The interpretation of any limiting integral curve passing through $\mathcal{S}$, is that $t$ is constant over any connected portion of that trajectory which lies within $\mathcal{S}$.

Summing up this analysis we can state the following

Theorem 6.1. The limiting system (6.3, 6.4) generates a dynamical system in the usual sense, except that for orbits passing through $\mathcal{S}$, the solution may be multiply defined, in the past as it enters, and in the future as it exits $\mathcal{S}$. Moreover, time is constant along a portion of any orbit entirely within $\mathcal{S}$, and for each orbit, the total time spent in $\mathcal{S}$ is of Lebesgue measure zero. Finally, for any open neighborhood $\mathcal{N}$ of $\mathcal{A}_{1}^{(\infty)}$, there exists $n_{\mathcal{N}}$ such that $\mathcal{A}_{1}^{(n)} \subset \mathcal{N}$ for all $n \geq n_{\mathcal{N}}$.

Remark 6.2. For the system $(6.3,6.4)$ we can define $\nu_{\text {eff }}^{\infty}$ in a similar way to (3.1). Indeed if $\Sigma\left(y_{1}\right)$ denotes the set of all solutions $\left(Y_{1}(s), t(s)\right)$ of (6.4) such that $Y_{1}(s) \in \mathcal{A}_{1}^{(\infty)}$ for all $s \in \mathbb{R}$, and $Y_{1}(0)=y_{1}$, then

$$
\frac{1}{\nu_{\text {eff }}^{\infty}\left(y_{1}\right)} \equiv \inf _{\left(Y_{1}(\cdot), t(\cdot)\right) \in \Sigma\left(y_{1}\right)} \sup _{-\infty<s<0} \frac{1}{|s|} \int_{s}^{0} \frac{\frac{\mathrm{d} t}{\mathrm{~d} \sigma}}{\nu_{1}^{(\infty)}\left(Y_{1}(\sigma)\right)} \mathrm{d} \sigma
$$




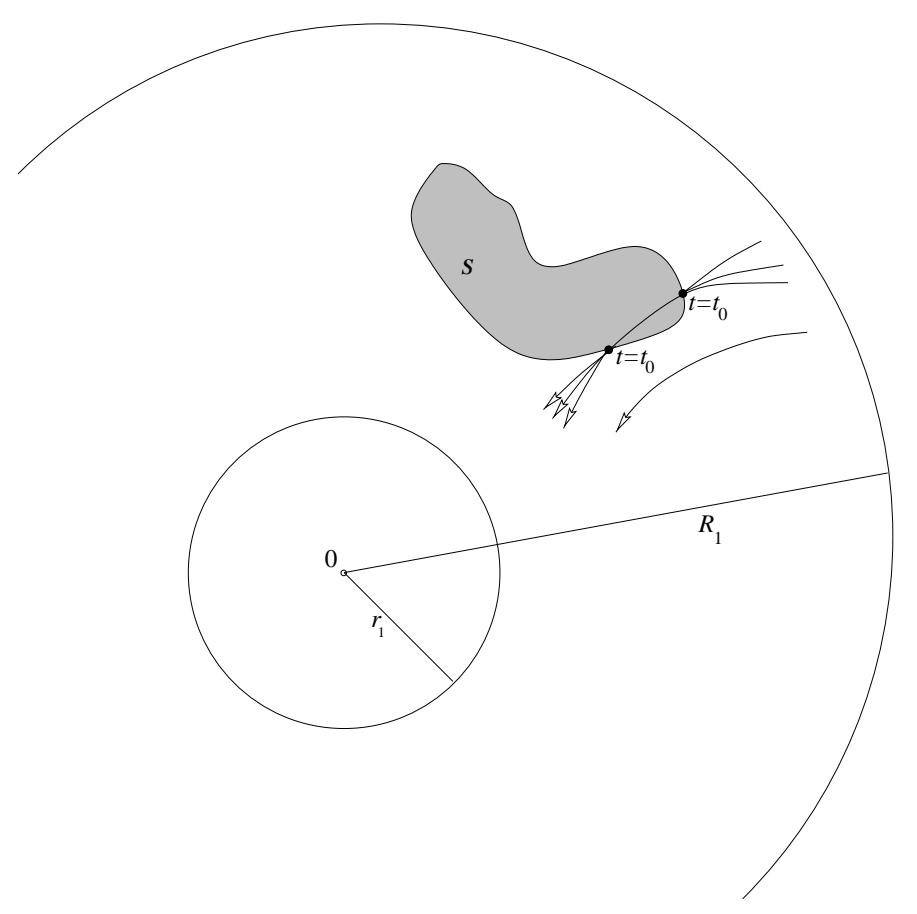

Figure 1. Possible behavior for limiting dynamical system.

makes sense. Then the analogue of Lemma 3.2 is still valid, that is

$$
\left\|y_{1}\right\| \leq \kappa_{0} G \frac{\nu^{2}}{\nu_{\mathrm{eff}}^{\infty}\left(y_{1}\right)} .
$$

In Remark 2.5 we observed that for large enough $\kappa_{1}$, the singular set $\mathcal{S}$ is empty. Under a similar assumption, we sketch in a final remark below how we can estimate the difference between solutions of the iterated system and the Navier-Stokes equations.

Remark 6.3. First note that if $\kappa_{1} / \kappa_{0} \gg 1$ then on the absorbing ball $\mathcal{B}_{\|\cdot\|}\left(0, R_{1}\right)$ we have

$$
\left\|y_{\infty}\right\|^{2} \leq(1+\epsilon)\left\|y_{1}\right\|^{2}
$$

hence

$$
\left\|y_{\infty}-y_{1}\right\|^{2}=\left\|Q_{1} y_{\infty}\right\|^{2}=\left\|y_{\infty}\right\|^{2}-\left\|y_{1}\right\|^{2}
$$

and $\tilde{\nu}_{1}^{(n)}$ defined in (1.13) satisfies

$$
0 \leq \tilde{\nu}_{1}^{(n)}\left(y_{1}\right)-\nu \leq \frac{c_{3}}{\nu \kappa_{0}^{2}} \epsilon\left\|y_{1}\right\|^{2}
$$

Indeed for (6.5), one need only choose $\bar{\xi}_{\epsilon}$ so that $g\left(\bar{\xi}_{\epsilon}\right)=\epsilon$, and use Lemma 2.3, while for (6.6) one may use (2.20) and notice that

$$
\tilde{\nu}_{1}^{(n)}\left(y_{1}\right)=\nu_{2}^{(n)}\left(y_{1}+z_{1}\right)
$$


A simple rearrangement of (1.12) gives

$$
\frac{\mathrm{d} y_{1}}{\mathrm{~d} t}+\nu A y_{1}+P_{1}\left[B\left(y_{1}\right)+B^{\prime}\left(y_{1}\right) z_{1}\right]=f-\left(\tilde{\nu}_{1}^{(n)}\left(y_{1}\right)-\nu\right) A y_{1},
$$

and therefore for $\kappa_{1}$ large enough, the solutions of (1.9) will stay near the solutions of

$$
\frac{\mathrm{d} y_{1}}{\mathrm{~d} t}+\nu A y_{1}+P_{1}\left[B\left(y_{1}\right)+B^{\prime}\left(y_{1}\right) z_{1}\right]=f,
$$

over finite time intervals. We complete the remark by recalling that was shown in [4] that for large enough $\kappa_{1}$, solutions of (6.7) stay near those of the Navier-Stokes equations over finite time intervals.

Acknowledgements. This work was partially supported by NSF grant number DMS-9706903. Much of this work was advanced while all three authors were visiting the Institute for Mathematics and its Applications at the University of Minnesota. OPM wishes to acknowledge the hospitality of the Department of Mathematics at Indiana University, as well as the use of some of the facilities at the National Institutes of Standards and Technology, where he is a guest worker.

\section{REFERENCES}

[1] P. Constantin and C. Foias, Navier-Stokes Equations, Univ. Chicago Press, Chicago, IL (1988).

[2] N. Dunford and J.T. Schwartz, Book Linear Operators, Wiley, New York (1958) Part II.

[3] C. Foias, What do the Navier-Stokes equations tell us about turbulence? in Harmonic analysis and nonlinear differential equations (Riverside, CA, 1995). Contemp. Math. 208 (1997) 151-180.

[4] C. Foias, O.P. Manley and R. Temam, Modelling of the interaction of small and large eddies in two-dimensional turbulent flows. RAIRO Modél. Math. Anal. Numér. 22 (1988) 93-118.

[5] C. Foias, O.P. Manley and R. Temam, Approximate inertial manifolds and effective viscosity in turbulent flows. Phys. Fluids A 3 (1991) 898-911.

[6] C. Foias, O.P. Manley and R. Temam, Iterated approximate inertial manifolds for Navier-Stokes equations in 2-D. J. Math. Anal. Appl. 178 (1994) 567-583.

[7] C. Foias, O.P. Manley, R. Temam and Y.M. Treve, Asymptotic analysis of the Navier-Stokes equations. Phys. D 9 (1983) $157-188$.

[8] C. Foias and B. Nicolaenko, On the algebra of the curl operator in the Navier-Stokes equations (in preparation).

[9] R.H. Kraichnan, Inertial ranges in two-dimensional turbulence. Phys. Fluids 10 (19671) 417-1423.

[10] W. Heisenberg, On the theory of statistical and isotropic turbulence. Proc. Roy. Soc. Lond. Ser. A. 195 (1948) $402-406$.

[11] E. Hopf, A mathematical example displaying features of turbulence. Comm. Appl. Math. 1 (1948) 303-322.

[12] R. Temam, Infinite-dimensional Dynamical Systems in Mechanics and Physics, 2nd edition, Springer-Verlag, New York (1997).

[13] T. von Karman, Tooling up mathematics for engineering. Quarterly Appl. Math. 1 (1943) 2-6. 